\title{
TABLE-BASED IDENTIFICATION PROTOCOL OF COMPUTATIONAL RFID TAGS
}

\author{
Yunmin Kim, Ji Hyoung Ahn, and Tae-Jin Lee \\ College of Information and Communication Engineering \\ Sungkyunkwan University, Suwon 16419, South Korea \\ kym0413@skku.edu, beramodedskku.edu, tjleedskku.edu
}

\begin{abstract}
Computation RFID (CRFID) expands the limit of traditional RFID by granting computational capability to RFID tags. RFID tags are powered by Radio-Frequency $(R F)$ energy harvesting. However, CRFID tags need extra energy and processing time than traditional RFID tags to sense the environment and generate sensing data. Therefore, Dynamic Framed Slotted ALOHA (DFSA) protocol for traditional RFID is no longer a best solution for CRFID systems. In this paper, we propose a table-based CRFID tag identification protocol considering CRFID operation. An RFID reader sends the message with the frame index. Using the frame index, CRFID tags calculate the energy requirement and processing time information to the reader along with data transmission. The RFID reader records the received information of tags into a table. After table recording is completed, the optimal frame size and proper time interval is provided based on the table. The proposed CRFID tag identification protocol is shown to enhance the identification rate and the delay via simulations.
\end{abstract}

\section{KEYWORDS}

Computational RFID, Energy harvesting, Sensing energy, Processing delay, Tag identification

\section{INTRODUCTION}

Internet of Things (IoT) is one of the emerging technologies that connects the physical world of things to the Internet [1]. Various applications such as metering, surveillance system, and factory maintenance systems are expected to be realized by IoT networks. There are many challenges to implement IoT networks [2]. IoT communications aim to support a massive number of deployed devices. To collect information of a large number of devices, efficient Medium Access Control (MAC) protocol is required. Moreover, there is an energy consumption issue due to energycritical small IoT devices. Devices should be energy efficient to maximize the network lifetime to provide better network maintenance and reliability.

Radio Frequency IDentification (RFID) is a contact-less identification technology. An RFID network consists of a RFID reader and multiple tags [3]. RFID tags classified into passive tags and active tags. Passive RFID tags transmit data by backscattering the signal of the reader. Since the transmission relies on the signal from the reader, passive tags are battery-less and semiDavid C. Wyld et al. (Eds) : NETCOM, NCS, WiMoNe, CSEIT, SPM - 2015 pp. 59-67, 2015. @ CS \& IT-CSCP 2015

DOI : $10.5121 /$ csit.2015.51606 
permanent. Traditional RFID tags can only send the predefined information such as unique IDs of tags. Thus, the role of RFID tags may be limited in IoT networks which require smarter and more complex jobs of devices.

Computational RFID (CRFID) is an enhanced RFID that can overcome the limitation of the traditional RFID. CRFID incorporating the sensing capability into RFID expands the functionality of RFID [4]. Fig. 1 indicates the simple concept of the CRFID tag. In a sensor part, a surrounding environment is measured and data is produced by local computing process. Then, the generated data is delivered to the RFID part and tags transmit it by backscattering the signal of the reader. With this computational capability, potential applicability of CRFID can be widened with sufficiently low energy consumption [5]. In this sense, CRFID is treated as a viable solution of enabling IoT networks [6].

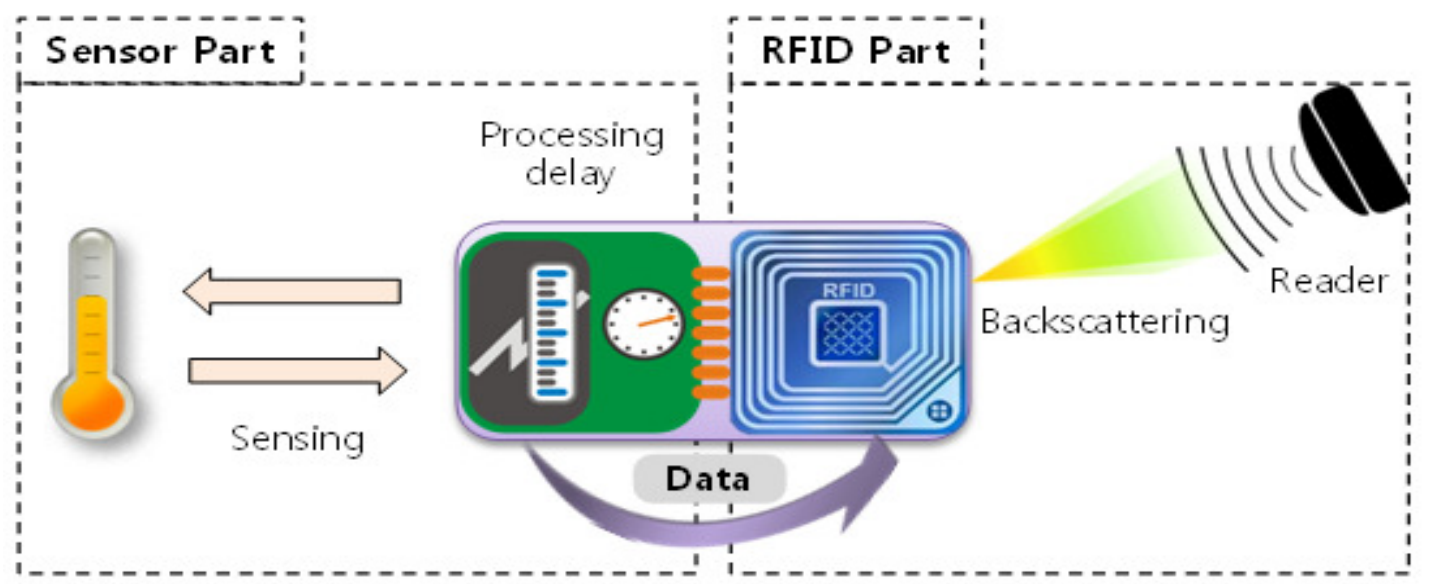

Figure 1. Structure of CRFID tag with sensing and processing capability.

There have been researches for CRFID systems. Yang et al. [7] proposed a switching mechanism of the firmware of a CRFID tag by the command from the reader. The inefficiency of firmware switching of a CRFID tag using wired interface is stated. To resolve the problem, the reader command for firmware switching is designed to be compatible with the EPC standard. The energy overhead and switching delay are simulated. In [8], an efficient way to transmit bulk data of a CRFID tag is studied. Authors stated the low performance of data stream of a CRFID tag caused by Cyclic Redundancy Check (CRC) calculation. Then, they proposed an efficient data transfer scheme for the CRFID tag by precomputing and exploiting the intermediate computations for CRC. Since the latency of CRFID tags is reduced with the proposed scheme, data transfer efficiency is improved. Wickramasinghe et al. [9] studied the ambulatory monitoring systems using CRFID sensors. Receiving data is segmented based on the natural activity boundaries and an algorithm to track the body movement transition is presented. In the experiment, CRFID in ambulatory monitoring shows high performance.

Existing studies do not tackle the most important difference of CRFID tags from traditional RFID tags. With the sensing operation, CRFID tags need extra energy and processing time before communicating with the reader. Dynamic Framed Slotted ALOHA (DFSA), which is used in the conventional RFID identification [10], may not be compatible with CRFID systems. Estimation of the number of tags in DFSA will no longer match with the actual number of active CRFID tags due to new characteristics of CRFID. Thus, the optimal frame size needs to be redesigned in DFSA. 
In this paper, we propose a CRFID tag identification protocol considering the sensing capability. Initially, a CRFID reader collects the energy and processing time requirement of individual tags along with the identification process. When the reader collects all of the energy requirements and processing time of tags, reader estimates the exact number of active tags at every frames. Then, the optimal frame size is decided using the table to maximize the identification rate.

\section{PROPOSED CRFID TAG IDENTIFICATION METHOD}

\subsection{DFSA Algorithm in CRFID Systems}

One of the key differences of the CRFID tag is that it senses and generates data to report. With this sensing and processing capability, CRFID tag requires extra energy and processing time for local computation. Furthermore, energy and processing time requirement among CRFID tags may vary with one another. Depending on the type of sensing job, some CRFID tags may need more energy than others. Received energy from the reader will also vary depend on the distance between the reader and the tag. Another difference of CRFID tag is continuous data transfer. While conventional RFID tags stop operating after the successful identification, CRFID tags continuously respond to the reader when they have data to transmit.

For the conventional RFID, DFSA algorithm is used for tag identification. In DFSA, a reader assigns a frame size, the number of time slots in a frame. Then, tags randomly select their own time slots and transmit their IDs to the reader. In [11], it is shown that the optimal frame size is the same as the number of identifying tags. The number of identifying tags is estimated using the fraction of idle slots (zero estimation) [12]. Since the DFSA algorithm does not reflect the novel characteristics of the CRFID tag, it cannot simply adapt to CRFID systems.

Fig. 2 shows the CRFID tag identification process using DFSA. We assume that the reader provides extra energy for sensing operation at the beginning of a frame. Tags 1,2, and 5 require charging twice for sensing, while tags 3 and 4 need charging only once. The reader assigns 5 slots in frame 1 since the number of tags is 5 . However, only tags 3 and 4 respond to the reader. Then, the reader assigns 3 slots to frame 2 by zero estimation. In frame 2, all the tags respond to the reader and contend to transmit data. Frame 2 cannot accommodate all the active tags since the number of active CRFID tags is larger than the frame size. Furthermore, because of the processing delay, tags actually contend in 2 slots which causes further degradation of performance.

\subsection{System Model}

Now, we describe the proposed table-based CRFID tag identification protocol. A CRFID network consists of a reader and multiple tags. Since the CRFID network is likely to be installed for a purpose, we assume that the reader knows the number of tags. The tags are equipped with small capacitors that can store relatively small amount of energy. At the beginning of every frame, the reader sends a packet intended for charging tags to support sensing operation. The number of required charging for sensing is randomly distributed in $\left[1, e_{\max }\right]$. The main idea of the proposed MAC protocol is to collect the energy and processing time requirements of tags during the tag identification. After that, the reader can schedule the optimal frame size to identify tags based on the table. Also, the time duration for sensing operation is provided based on the table. The proposed scheme can be divided into table recording phase and normal phase. 


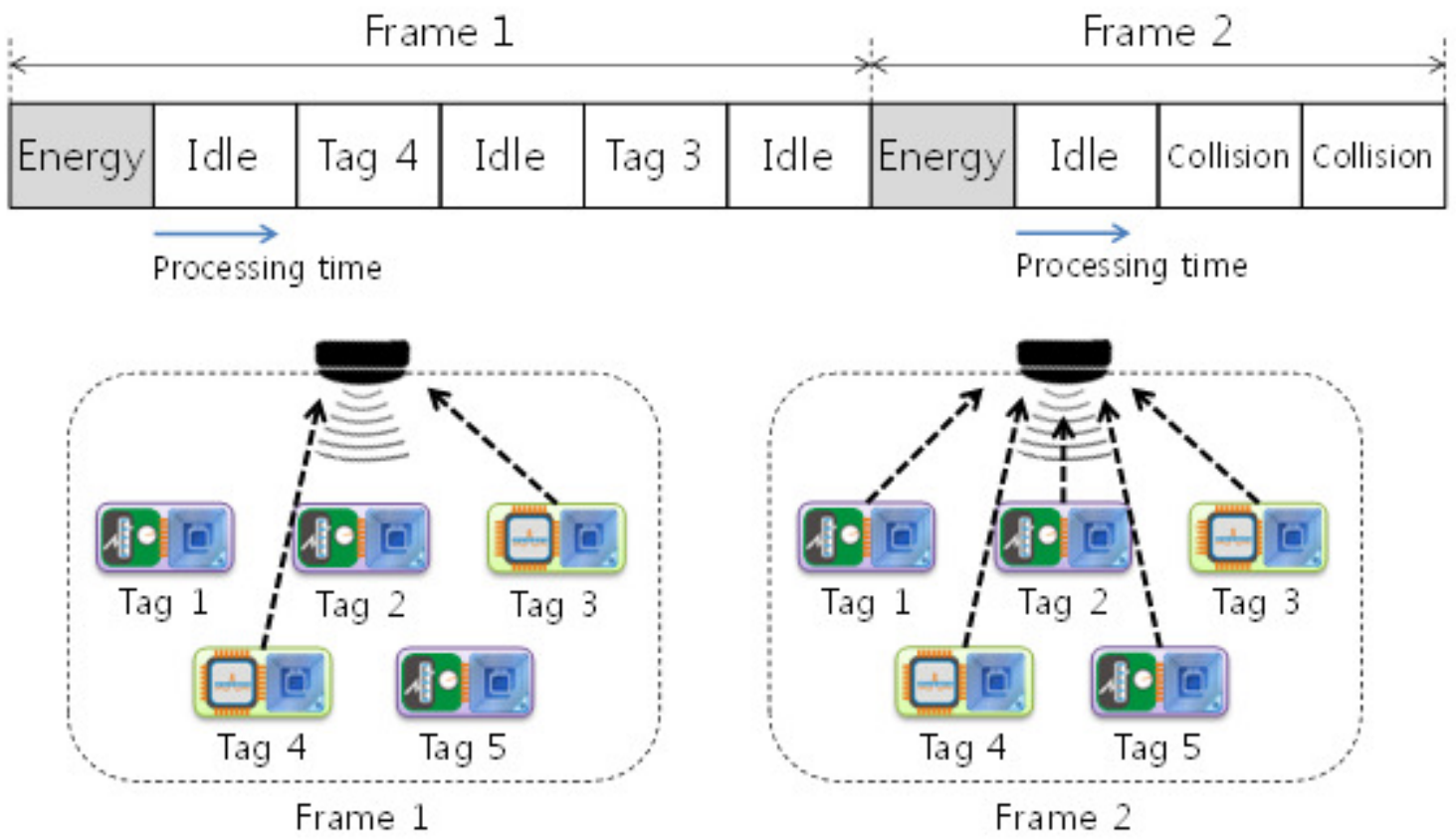

Figure 2. CRFID tag identification using DFSA algorithm.

\subsection{Table Recording Phase}

When the reader starts to receive data, it operates as the table recording phase. In the table recording phase, the reader collects the energy requirement $(e)$ and processing delay $(t)$ of individual tag along with receiving data. Let $e_{i}$ and $t_{i}$ be the energy and processing time requirements of tag $i$. Since energy and processing the information of tags is yet to be collected, the reader decides frame as the number of entire tags. The reader sends the querying message with a frame index. When the tag $i$ charges sufficient enough amount of energy for sensing, it performs sensing and records the frame index as its $e_{i}$. Then, the tag transmits $e_{i}$ and $t_{i}$ along with the generated data to the reader. The reader records the received $e_{i}$ and $t_{i}$ information in the table.

Fig. 3 shows an example of collecting process for energy and processing information in the table recording phase. In frame 1, tags 3 and 4 respond to the reader and consume the charged energy. Since other tags need more energy to perform sensing, they sleep in frame 1 . The reader records $e_{3}, t_{3}, e_{4}$, and $t_{4}$ of tags 3 and 4 in the table. In frame 2, all tags become active and respond to the reader. Since only tag 1 succeeds in frame 2 , the reader obtains $e_{1}$ and $t_{1}$. Collided tags 2 and 5 may send their $e$ and $t$ information in the subsequent frames. The table recording phase ends when all the information of $e$ and $t$ are completely collected. 


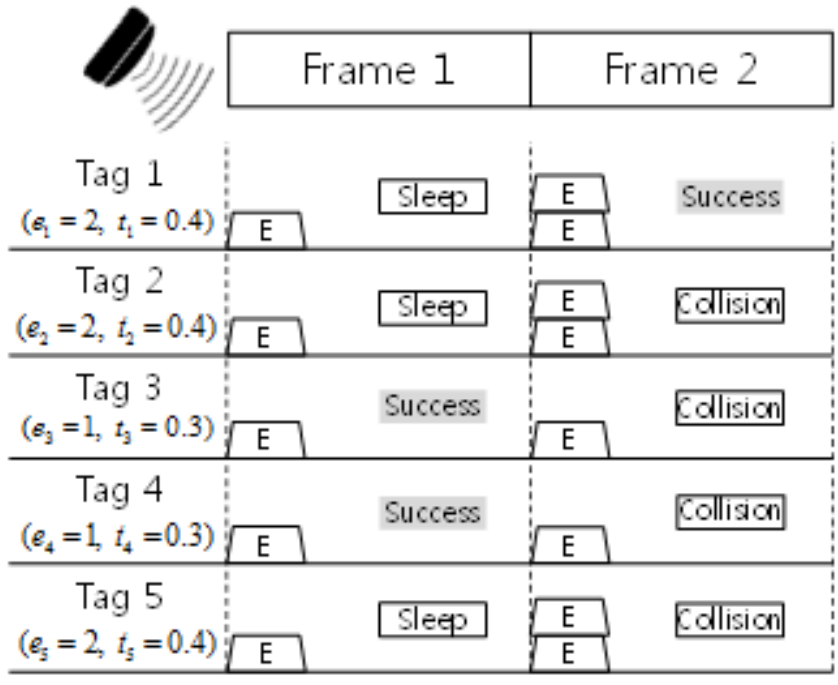

Table
\begin{tabular}{|c|c|c|c|c|c|}
\hline Tag ID & 1 & 2 & 3 & 4 & 5 \\
\hline$e_{i}$ & - & - & 1 & 1 & - \\
\hline$t_{i}$ & - & - & 0.3 & 0.3 & - \\
\hline
\end{tabular}

Frame 1

Table
\begin{tabular}{|c|c|c|c|c|c|}
\hline Tag ID & 1 & 2 & 3 & 4 & 5 \\
\hline$e_{i}$ & 2 & - & 1 & 1 & - \\
\hline$t_{i}$ & 0.4 & - & 0.3 & 0.3 & - \\
\hline
\end{tabular}

Frame 2

Figure 3. An example of the table recording process.

\subsection{Normal Phase}

Once the table is filled with all the information of $e$ and $t$, the reader moves to the normal phase. In the normal phase, the reader can decide which tags try to transmit in the current frame. If $e_{i}$ of tag $i$ is a divisor of the current frame index, the tag will become active and transmit data. To maximize the identification rate, the reader should set the frame size to be the number of the active tags. By searching for the table, the reader evaluates the $i$-th frame size as

$$
L_{i}=\sum_{j=1}^{N_{\text {tag }}} 1_{\{0\}}\left(i \bmod e_{j}\right),
$$

where $1_{\{0\}}$ and $N_{\text {tag }}$ refer to the indicator function and the number of tags.

The reader can assign the time interval to mitigate the processing delay of tags. To ensure the processing delay of tags to be tolerable, the reader chooses the maximum $t$ among those of the active tags in the frame. Then, the processing interval for the $i$-th frame is decided as

$$
T_{i}=\max _{j \in\left\{l l 1_{\{0\}}\left(i \bmod e_{j}\right)=1\right\}}\left(t_{j}\right) .
$$

Fig. 4 shows the 9th and 10th frame structures in the normal phase. In the normal phase, the processing interval of length $T_{i}$ is assigned after the energy transfer from the reader. So, all tags can sense and produce data before the slot contention. We assume that the information for energy and processing time of all tags are collected before frame 9 . In frame 9, the reader finds out that tags 3 and 4 will be active tags by the table with the frame index 9 . Then, the frame size is determined as $L_{9}=2$. The processing time interval for frame 9 is $T_{9}=0.3$ since $t_{3}=t_{4}=0.3$ in the table. In frame 10, all tags will be active since 1 and 2 are divisors of the frame index 10. 
The frame size $L_{10}$ is decided as 5 . Then, the processing time interval of frame 10 becomes $T_{10}=0.4$.

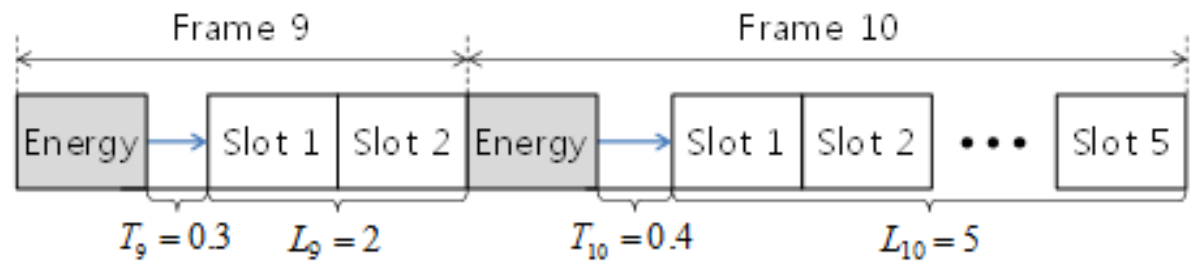

\begin{tabular}{|c|c|c|c|c|c|}
\multicolumn{9}{|c}{ Table } & $\begin{array}{l}L_{9}=\sum_{j=1}^{5} 1_{\{0\}}\left(10 \bmod e_{j}\right)=2 \\
T_{9}=\max _{j \in\{3,4\}}\left(t_{j}\right)=0.3\end{array}$ \\
\hline$e_{i}$ & 2 & 2 & 1 & 1 & 2 \\
\hline$t_{i}$ & 0.4 & 0.4 & 0.3 & 0.3 & 0.4 \\
&
\end{tabular}$\quad \begin{aligned} & L_{10}=\sum_{j=1}^{5} 1_{\{0\}}\left(10 \bmod e_{j}\right)=5 \\
& T_{10}=\max _{j \in\{1,2,3,4,5\}}\left(t_{j}\right)=0.4\end{aligned}$

Figure 4. The $9^{\text {th }}$ and $10^{\text {th }}$ frames in the normal phase.

\section{Performance Evaluation}

In this section, we evaluate the performance of our proposed CRFID tag identification method. The identification rate at frames and the delay are evaluated for varying number of CRFID tags. Monte Carlo method with MATLAB as a simulation tool is used. For each iteration, tags uniformly distributed in a square area with a reader located in the center. Then, tags perform random contention to send data to the reader using FSA or the proposed protocol. Since DFSA is not feasible as shown in Fig. 2, we compare the proposed method with the Framed Slotted ALOHA (FSA). The frame size of FSA is fixed as a half of the number of existing tags considering energy requirement. Energy requirement of CRFID tags is determined by the distance from the reader. During the simulation, the identification rate in frames and the delay of each tag are recorded. The parameters used in the simulations are shown in Table I.

Table 1. Simulation parameters.

\begin{tabular}{|l|l|}
\hline \multicolumn{1}{|c|}{ Parameter } & \multicolumn{1}{c|}{ Value } \\
\hline Simulation area & $5 \mathrm{~m} \times 5 \mathrm{~m}$ \\
\hline Number of tags & $200 \sim 300$ \\
\hline Maximum energy requirement & 3,6 \\
\hline Processing delay & $0.4 \mathrm{~ms}$ \\
\hline Duration of time slot & $1 \mathrm{~ms}$ \\
\hline Data size & $96 \mathrm{bits}$ \\
\hline
\end{tabular}

Fig. 5 shows the identification rate as frames evolve. Since the number of active CRFID tags changes periodically, the identification rate fluctuates for a fixed frame size in FSA. In the 
proposed scheme, the identification rate fluctuates at first, then it converges to the value higher than that of the legacy FSA. The identification rate is maximized since the optimal frame size is allocated using the table. As the maximum energy requirement increases, the number of active tags at frames becomes smaller. Then, the identification rate decreases since the fixed frame size becomes too large. Also, the convergence of the identification time in the proposed method for larger minimum energy requirement becomes longer since the table information is collected more slowly. The proposed scheme shows better performance than the legacy FSA after about 60 frames.
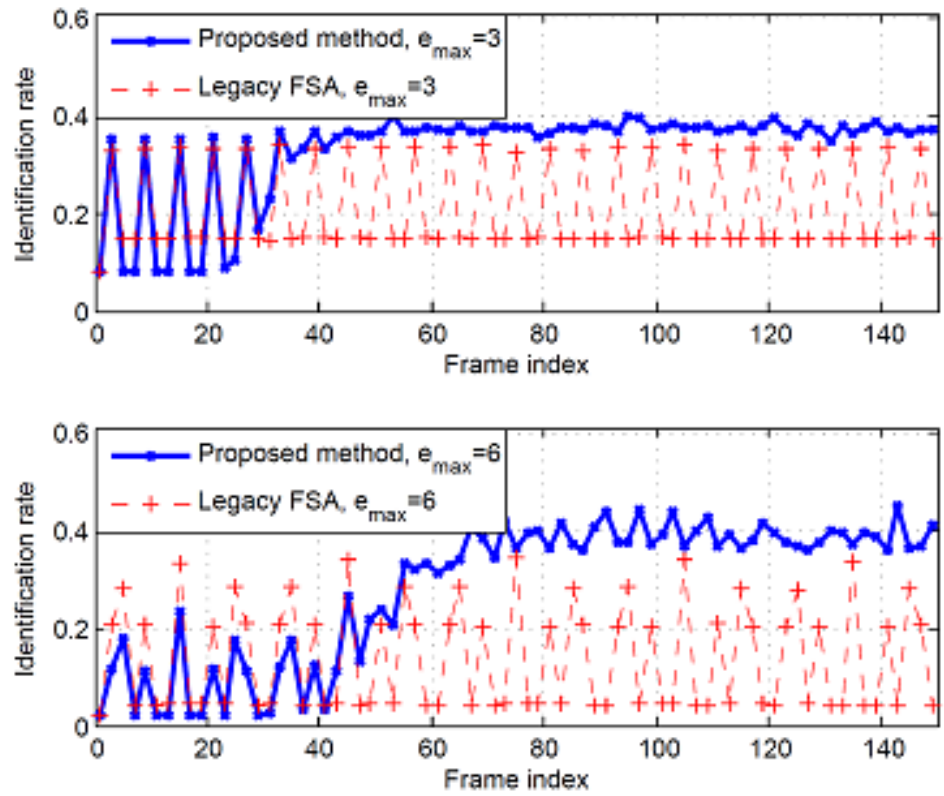

Figure 5. Identification rate of CRFID systems as frame evolve.

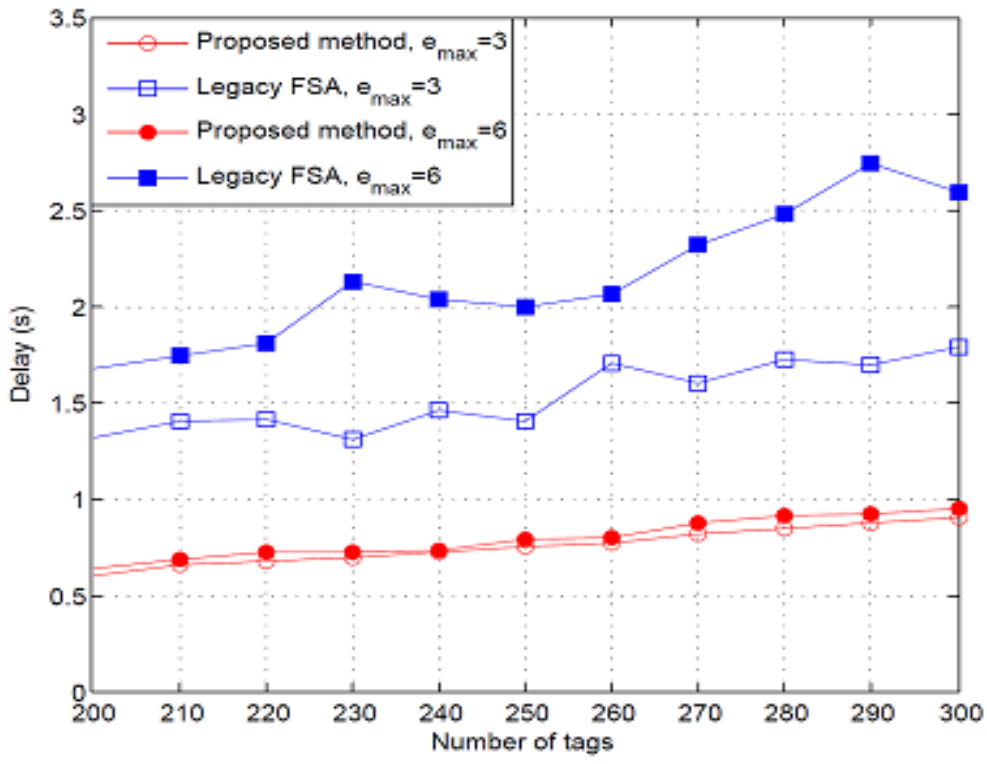

Figure 6. Delay of CRFID tags for varying number of tags. 
Fig. 6 shows the delay of CRFID tags for varying number of tags. The average delay of tags increases as the number of tags increases. Since the chance of transmission reduces as the number of tags increases, the delay of tags becomes larger. The proposed method shows lower delay than the conventional FSA. By maximizing the identification rate, the delay between transmissions is reduced in the proposed scheme. When the maximum energy requirement is 6 , the delay is larger than that of the case with the energy requirement of 3 . Since tags sleep longer when more collisions occur, the delay increases. In the proposed method, the delay increment is negligible compared to that of the legacy FSA. Since the optimal frame size is reduced the chance of collisions, delay increment caused by sleep interval is minimized.

\section{CONCLUSIONS}

In this paper, we have proposed a CRFID tag identification protocol considering the features of CRFID tags. Especially, we consider CRFID tags which need energy for sensing process. The proposed MAC protocol collects the energy and processing time requirements of tags. After the requirement of all tags is collected, the reader can schedule the optimal frame size. We also provide the time duration to support the processing delay of CRFID tags in every frame. We have shown that the proposed method can improve the identification rate and delay compared to the conventional FSA scheme.

\section{ACKNOWLEDGEMENTS}

This work was supported by the National Research Foundation of Korea (NRF) grant funded by the Korean government (MSIP)(2014R1A5A1011478, 2015R1A2A2A01004067).

\section{REFERENCES}

[1] M. Bolic, M. Rostamian, and P. M. Djuric, "Proximity Detection with RFID: A Step Toward the Internet of Things," IEEE transactions on Pervasive Computing, vol. 14, no. 2, pp. 70-76, Jun. 2015.

[2] H.-D. Ma, "Internet of Things: Objectives and Scientific Challenges," Springer Journal of Computer Science and Technology, vol. 26, no. 6, pp. 919-924, Nov. 2011.

[3] C. W. Park, J. H. Ahn, and T.-J. Lee, "RFID Identification Protocol with Optimal Frame Size for Varying Slot Time," International Journal of Information and Electronics Engineering, vol. 4, no. 2, pp. 87-91, Mar. 2014.

[4] A. P. Sample, D. J. Yeager, P. S. Powledge, A. V. Mamishev, and J. R. Smith, "Design of an RFIDBased Battery-Free Programmable Sensing Platform," IEEE Transactions on Instrumentation and Measurement, vol. 57, no. 11, pp. 2608-2615, Nov. 2008.

[5] B. Sheng and C. C. Tan, "Group Authentication in Heterogeneous RFID Networks," in Proc. IEEE Conference on Technologies for Homeland Security, pp. 167-172, Nov. 2015.

[6] L. Atzori, A. Iera, and G. Morabito, "The Internet of Things: A survey," International Journal of Computer and Telecommunications Networking, vol. 54, no. 15, pp. 2787-2805, Oct. 2010.

[7] W. Yang, D. Wu, M. J. Hussain, and L. Liu, "Wireless Firmware Execution Control in Compuational RFID Systems," in Proc. of IEEE International Conference on RFID, pp. 129-136, Apr. 2015. 
[8] Y. Zheng and M. Li, "Read Bulk Data from Computational RFID," in Proc. of IEEE INFOCOM, pp. 495-503, Apr. 2014.

[9] A. Wickramasinghe and D. C. Ranasinghe, "Ambulatory Monitoring Using Passive Computational RFID Sensors,” IEEE Transactions on Sensor Journals, vol. 15, no. 10, pp. 5859-5869, Oct. 2015.

[10] "EPC Radio-Frequency Identification Protocols Generation-2 UHF RFID Protocol for Communications at 860MHz-960MHz," Version 2.0.0, EPCglobal, Nov. 2013.

[11] J.-R. Cha and J.-H. Kim, "Novel Anti-collision Algorithms for Fast Object Identification in RFID Systems," in Proc. of International Conference on Parallel and Distributed Systems, pp. 63-67, Jul. 2005.

[12] G. Khandelwal, K. Lee, A. Yener, and S. Serbetli, "ASAP: A MAC Protocol for Dense and Timeconstrained RFID Systems," EURASIP Journal on Wireless Communications and Networking, vol. 2007, no. 2, pp. 1-13, Jan. 2007.

\section{AUTHORS}

Yunmin Kim received the B.S. and M.S. degrees in electrical and computer engineering from Sungkyunkwan University, Korea, in 2012 and 2014, respectively. $\mathrm{He}$ is currently working toward the Ph.D. degree in electrical and computer engineering at Sungkyunkwan University since March 2014. His research interests include energy-harvesting networks, cognitive radio networks, vehicular networks, wireless LANs, RFID/NFC, and IoT, resource allocation and Medium Access Control (MAC) of wireless communication networks.

Ji Hyoung Ahn received the B.S. and M.S. degrees in electronic, electrical, and computer engineering from Sungkyunkwan University, Korea, in 2010 and 2012, respectively. He is currently working toward the Ph.D. degree in the College of Information and Communication Engineering at Sungkyunkwan University since March 2012. His research interests include routing protocols for ad hoc networks, wireless communication networks, wireless LAN, and wireless PAN.

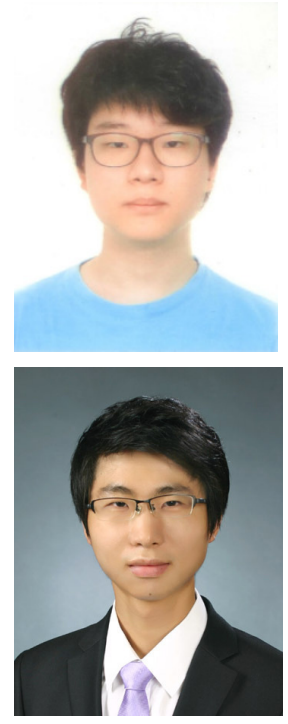

Tae-Jin Lee received his B.S. and M.S. in electronics engineering from Yonsei University, Korea in 1989 and 1991, respectively, and the M.S.E. degree in electrical engineering and computer science from University of Michigan, Ann Arbor, in 1995. $\mathrm{He}$ received the Ph.D. degree in electrical and computer engineering from the University of Texas, Austin, in May 1999. In 1999, he joined Corporate R \& D Center, Samsung Electronics where he was a senior engineer. Since 2001, he has been Professor in the College of Information and Communication Engineering at Sungkyunkwan University, Korea. He was a visiting professor in Pennsylvania State University from 2007 to 2008. His research interests include performance evaluation,

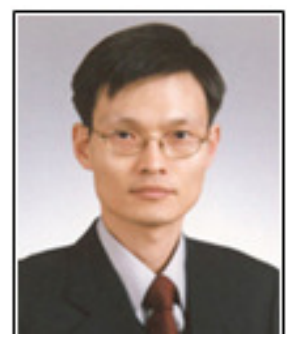
resource allocation, Medium Access Control (MAC), and design of communication networks and systems, wireless LANs/PANs, vehicular networks, energy-harvesting networks, IoT, ad hoc/sensor/RFID networks, and next generation wireless communication systems. He has been a voting member of IEEE 802.11 WLAN Working Group, and is a member of IEEE and IEICE. 